\title{
Non-linear Analysis of Models for Biological Pattern Formation: Application to Ocular Dominance Stripes
}

\author{
M. J. Lyons and L. G. Harrison \\ Physics Dept. (MJL) and Chemistry Dept. (LGH) \\ University of British Columbia \\ Vancouver, BC, Canada V6T 2A6 \\ e-mail: lyons@physics.ubc.ca
}

\begin{abstract}
We present a technique for the analysis of pattern formation by a class of models for the formation of ocular dominance stripes in the striate cortex of some mammals. The method, which employs the adiabatic approximation to derive a set of ordinary differential equations for patterning modes, has been successfully applied to reaction-diffusion models for striped patterns [1]. Models of ocular dominance stripes have been studied $[2,3]$ by computation, or by linearization of the model equations. These techniques do not provide a rationale for the origin of the stripes. We show here that stripe formation is a non-linear property of the models. Our analysis indicates that stripe selection is closely linked to a property in the dynamics of the models which arises from a symmetry between ipsilateral and contralateral synapses to the visual cortex of a given hemisphere.
\end{abstract}

\section{Introduction}

Much of biological pattern formation occurs in two-dimensional domains, such as single sheets of cells, surfaces of large single cells, or single layers of many nuclei within a single cell, such as the widely-studied Drosophila blastoderm. In many instances, the patterns formed are in essence a set of parallel stripes. For such cases as mammalian coat markings (e.g. zebra), while the general nature of the striped pattern may serve a biological purpose, a fair amount of variation from individual to individual may be tolerable in both the total number of stripes, their precise orientation, and the occurrence of defects such as branching. In other cases, such as the onset of insect segmentation, a pattern 
of a precisely-determined number of stripes, properly oriented and with no tendencies to break into spots or to form branches, is essential to development of the body plan. Most mutantions which disturb this pattern are lethal at an early developmental stage. Our work on striped patterns had insect segmentation as its initial motivation.

The mechanism of stripe formation remains in all cases unknown. Theoreticians have found reaction-diffusion theory, as initiated by Turing [12], a promising possibility. There are, however, some instances in which the mechanism is likely to be quite different, though the morphological phenomenon is the same. A well-known example of this kind is the formation of ocular dominance stripes. These are spatial patterns of synaptic connections observed in the striate cortex of some mammals, especially primates. In these highest vertebrates (and in contrast to some lower ones) each side of the brain receives roughly equal numbers of connections from both eyes. A stripe consists of cortical cells (in layer IVc) all of which receive connections from the same eye. The stripes bear a strong similarity to zebra stripes.

Ocular dominance patterns also closely resemble the striped patterns which we obtained by computation using a particular class of reaction-diffusion models [1]. This class has a particular dynamical symmetry. In addition to the computations, we showed analytically, using the adiabatic approximation, that this particular symmetry should generate stripes. Here, we apply the same technique to the ocular dominance models.

\section{Ocular Dominance Models}

Two well known models for OD patterns $[2,3]$ take the form of integro-differential equations. We consider a phenomenological model originally proposed by Swindale [2], based on a mechanism suggested by Hubel and Wiesel [4]. The densities of left and right synapses $n_{L}(\vec{r}, t)$ and $n_{R}(\vec{r}, t)$, which are functions of a two dimensional spatial coordinate $\vec{r}$ on the cortex and time, develop according to:

$$
\begin{aligned}
\frac{\partial n_{R}}{\partial t} & =f\left(n_{R}\right) \int\left(n_{R}\left(\vec{r}-\vec{r}^{\prime}\right) w_{R R}\left(\vec{r}^{\prime}\right)+n_{L}\left(\vec{r}-\vec{r}^{\prime}\right) w_{L R}\left(\vec{r}^{\prime}\right)\right) d \vec{r}^{\prime} \\
\frac{\partial n_{L}}{\partial t} & =f\left(n_{L}\right) \int\left(n_{R}\left(\vec{r}-\vec{r}^{\prime}\right) w_{R L}\left(\vec{r}^{\prime}\right)+n_{L}\left(\vec{r}-\vec{r}^{\prime}\right) w_{L L}\left(\vec{r}^{\prime}\right)\right) d \vec{r}^{\prime}
\end{aligned}
$$

The integrals sum competitive and cooperative interactions between like and opposite terminals over the entire patterned region, weighted by the intracortical interactions $w\left(\vec{r}^{\prime}\right)$ (shown in Figure 1). The function $f(n)$ takes into account the non-linear effects limiting the growth of the synaptic densities. We take a simple logistic form $f(n)=n(N-n)$ where $\mathrm{N}$ is the maximum density of synapses. Equations $(1,2)$ may be reduced to a single equation for the difference left and right densities with the assumption that the total density of synapses 
$N=n_{L}+n_{R}$ is constant [2]:

$$
\frac{\partial a}{\partial t}=(a * w+K)(N-a)(N+a) .
$$

$a=n_{R}-n_{L}, w=1 / 4\left(w_{R R}+w_{L L}\right), K=(N / 4) *\left(w_{R R}-w_{L L}\right)$ and $*$ indicates convolution. (3) represents a minimal model for OD stripes which has been shown by computation to possess the essential patterning capabilities exhibited by more complex models $[2,3,5]$.

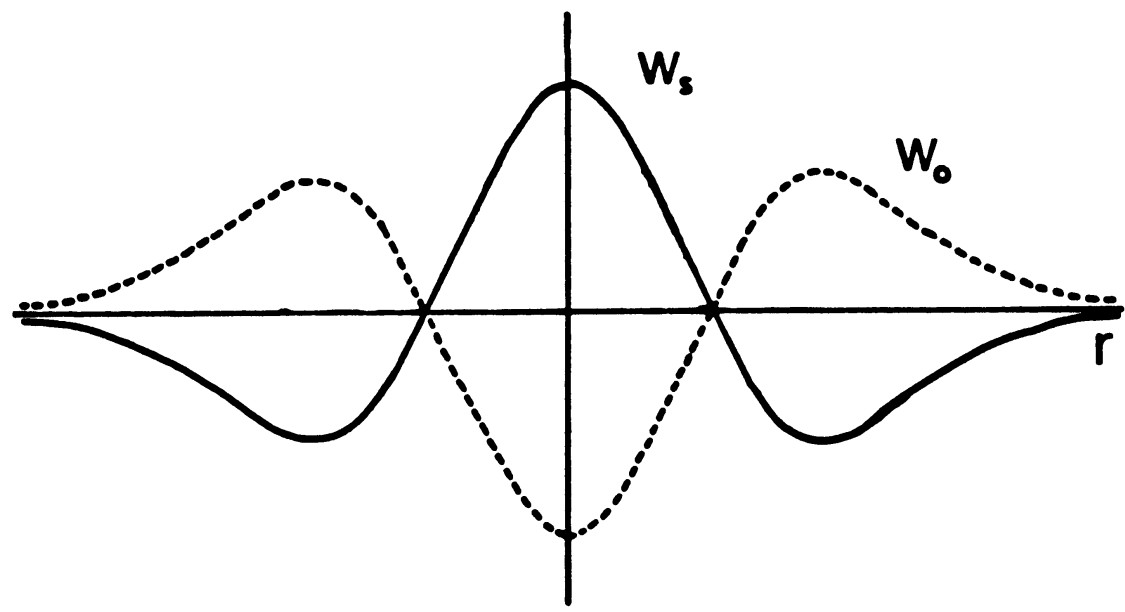

Figure 1 Shows typical weighting functions (kernels) for interactions between like synapses $\left(w_{0}=w_{R R}, w_{L L}\right.$, solid line) as well as between terminals from opposite eyes $\left(w_{0}=w_{L R}, w_{R L}\right.$, broken line). Pattern formation in this model results from short range activation and long range inhibition of like synapses, and vice-versa for the cross interactions (after [2]). For constant total density of synapses $N=n_{L}+n_{R}, w_{s}=-w_{o}[2]$.

\section{Non-linear Analysis}

We are primarily interested in models with symmetry between left and right terminals, i.e. $w_{R R}=w_{L L}$ so $K=0$. This implies that equation (3) is unchanged under the operation $a \rightarrow-a$. We analyze the simplest possible pattern-forming system having this symmetry:

$$
\frac{\partial a}{\partial t}=a * w-a^{3} .
$$

The coefficient of the cubic term can be set to unity without loss of generality by changing the dimensions of the ocular dominance $a$. Other constants may 
be absorbed into $w$. (4) has homogeneous steady state solution given by $a=0$ which corresponds to an unpatterned state consisting of an equal mixture of left and right terminals. Close to this state the linear part of the equation predominates:

$$
\frac{\partial a}{\partial t}=a * w .
$$

Equation (5) is exactly soluble by Fourier analysis. For the moment, we treat a finite domain, but otherwise leave the boundary conditions unspecified. The general solution is a superposition of plane waves with amplitudes $\xi_{k}(t)$

$$
\begin{aligned}
a(\vec{r}, t) & =\sum_{\vec{k}} \xi_{k}(t) e^{i \vec{k} \cdot \vec{r}}, \\
\xi_{\vec{k}, t} & =\xi_{0}(\vec{k}) e^{W(k) t} .
\end{aligned}
$$

$W(k)$ is the Fourier tranform of the kernel $w(r)$ which depends only on the magnitude of $\vec{k}$, and the $\xi_{0}(\vec{k})$ are to be determined from the initial condition.

To obtain an approximate solution to the nonlinear equations (4) we continue to write the solution as a sum of plane waves (6), and derive differential equations for the mode amplitudes. Substituting (6) into (4), multiplying on the left by $e^{i \vec{k} \cdot \vec{r}}$ and integrating over the domain using the orthogonality of plane waves:

$$
\frac{1}{A} \int e^{i\left(\vec{k}-\vec{k}^{\prime}\right) \cdot \vec{r}} d A=\delta_{\vec{k}, \vec{k}}, A=\text { domain area },
$$

we obtain the mode amplitude equations:

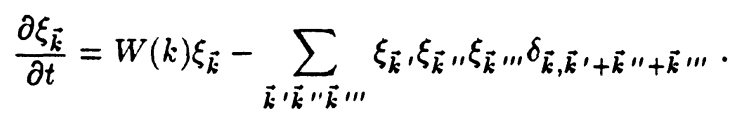

A similar set of equations has been derived for reaction-diffusion models of pattern formation [1]. Equation (9) is equivalent to the original form (4), and is intractable. It is possible to understand some of the behaviour of model (4) by studying it with a special form for $w(r)$ which allows an approximate solution of equations (9) by the adiabatic method. Briefly, the conditions for the approximation to hold are:

$$
\begin{aligned}
W\left(k_{c}\right) & >0 \text { for one value of } k_{c}, \\
W(k) & <0 \text { for } k \neq k_{c}, \\
|W(k)| & \gg\left|W\left(k_{c}\right)\right| .
\end{aligned}
$$

If these conditions are satisfied then the relaxation times of the stable modes are much shorter than that for the unstable modes. The stable mode amplitudes then adjust rapidly to the current values of the $\boldsymbol{\xi}_{\boldsymbol{k}_{\boldsymbol{c}}}$. In the adiabatic approximation [6] all of the stable modes $\left(k \neq k_{c}\right)$ are then eliminated from the 
dynamics leaving a set of ODEs for the unstable mode amplitudes, $k=k_{c}$ at all possible relative orientations $\phi$ :

$$
\dot{\xi}_{\phi}=W\left(k_{c}\right) \xi_{\phi}-3 \xi_{\phi} \xi_{\phi}^{*} \xi_{\phi}-6 \sum_{\phi^{\prime}} \xi_{\phi} \xi_{\phi^{\prime}}^{*} \xi_{\phi^{\prime}}, 0<\phi, \phi^{\prime}<\pi, \phi^{\prime} \neq \phi .
$$

For details on the derivation of equation (13) from (9) we suggest the reader consults [1], where a similar equation was found for a class of reaction-diffusion models which make striped patterns (see Figure 2).

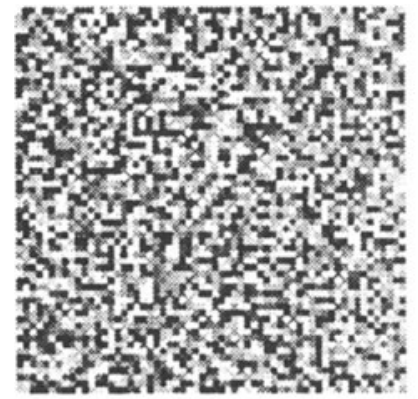

(a) $\mathrm{t}=0$

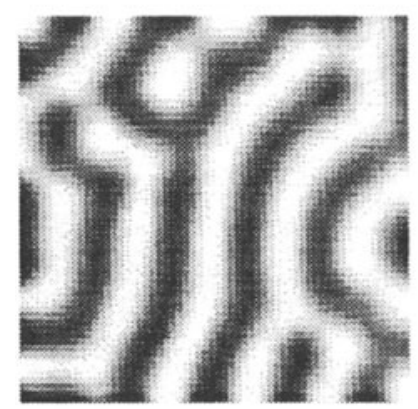

(c) $\mathrm{t}=5000$

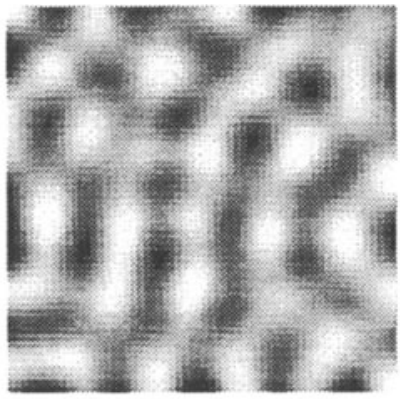

(b) $t=3000$

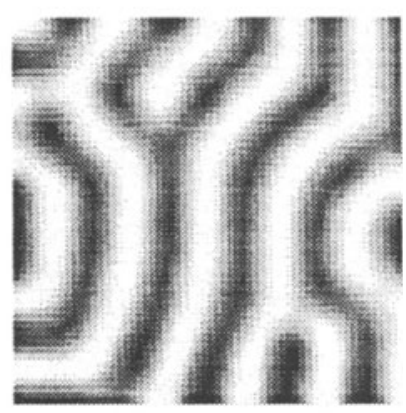

(d) $t=15000$

Figure 2 Time evolution of the concentration profile for one of the morphogens in a reaction-diffusion system with the same dynamical symmetry as equation $(4)[1,7,8]$. Times are iterations of a finite-difference algorithm. The domain is square with no-flux boundary conditions to mimic the behaviour observed at the boundary of area 17 of the cortex where OD stripes run perpendicular to the edge. The similarity of the RD patterns to OD stripes led us to investigate the properties of OD models. 
It may be shown for any number of unstable modes (same $k_{c}$, variable $\phi)$ that the only stable solutions of (13) are stripes [1]. We illustrate this for the simple case of a square domain where there are only four unstable modes corresponding to striped patterns in mutually perpendicular directions. The unstable modes have $\phi=0, \pi / 2, \pi, 3 \pi / 2$ and $k_{c}=2 \pi l_{c}, l_{c} \in Z^{+}$This constrains $l_{c}^{2}$ to integers which cannot be written as the sum of two squares. Then eqns. (13) are:

$$
\begin{aligned}
\dot{\xi}_{0} & =W \xi_{0}-3 \xi_{0} \xi_{0}^{*} \xi_{0}-6 \xi_{0} \xi_{\frac{\pi}{2}}^{*} \xi_{\frac{\pi}{2}} \\
\dot{\xi}_{\frac{\pi}{2}} & =W \xi_{\frac{\pi}{2}}-3 \xi_{\frac{\pi}{2}} \xi_{\frac{\pi}{2}}^{*} \xi_{\frac{\pi}{2}}-6 \xi_{\frac{\pi}{2}} \xi_{0}^{*} \xi_{0} .
\end{aligned}
$$

Eqn (18) has fixed points:

$$
\begin{gathered}
\text { (I) } \xi_{0}^{*} \xi_{0}=W / 3, \xi_{\frac{\pi}{2}}=0 \text { or } \xi_{\frac{\pi}{2}}^{*} \xi_{\frac{\pi}{2}}=W / 3, \xi_{0}=0 \\
\text { (II) } \xi_{0}^{*} \xi_{0}=\xi_{\frac{\pi}{2}}^{*} \xi_{\frac{\pi}{2}}=W / 9
\end{gathered}
$$

The linear stability matrix $A_{i j}=\partial \dot{\xi}_{i} / \partial \xi_{j}$ is:

$$
\mathbf{A}=\left(\begin{array}{cc}
W-6\left(\xi_{0}^{*} \xi_{0}+\xi_{\frac{\pi}{2}}^{*} \xi_{\frac{\pi}{2}}\right) & -6 \xi_{0} \xi_{\frac{\pi}{2}}^{*} \\
-6 \xi_{\frac{\pi}{2}} \xi_{0}^{*} & W-6\left(\xi_{0}^{*} \xi_{0}+\xi_{\frac{\pi}{2}}^{*} \xi_{\frac{\pi}{2}}\right)
\end{array}\right)
$$

evaluated at the fixed points. For solution (II), $\mathbf{A}$ has a negative and a positive eigenvalue indicating a saddle point. With solution (I) we find two negative eigenvalues, so the only stable pattern is a set of stripes, in either direction $\phi=0$ or $\pi / 2$. Mode suppression arises as a result of 'competitive'interactions.

\section{Discussion}

In the macaque monkey, ipsilateral and contralateral afferents to the cortex (i.e. connections from right or left eye to the same or the opposite side of the brain) are approximately equal in numbers. Also, left and right dominance stripes in layer IVc are similar in form and width (Figure 3(a)). The only difference between left and right dominated bands appears to be their connection to different eyes. This symmetry between ipsilateral and contralateral inputs is related to the selection of stripes rather than any other spatial pattern. In monocular deprivation experiments, this symmetry is disrupted, leading to a spotted pattern of ocular dominance in which terminals from the eye receiving visual information are more numerous than ones from the blindfolded eye. Binocular deprivation restores the left/right symmetry and stripes are observed when neither eye receives input during development.

In the cat, there is normally a quantitative asymmetry between the ipsilateral (about 30\%) and cortralateral (about 70\%) inputs to the cortex [9]. A 
splotchy pattern of ocular dominance is found (Figure 3(b)). Binocular deprivation gives a pattern quite unlike stripes. To examine the effect on ocular dominance patterns of varying degrees of asymmetry we suggest a comparative survey of various species of mammals with different ratios of ipsilateral/contralateral afferents.

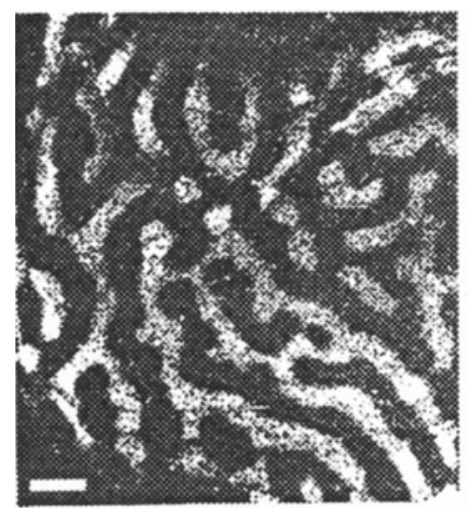

(a)

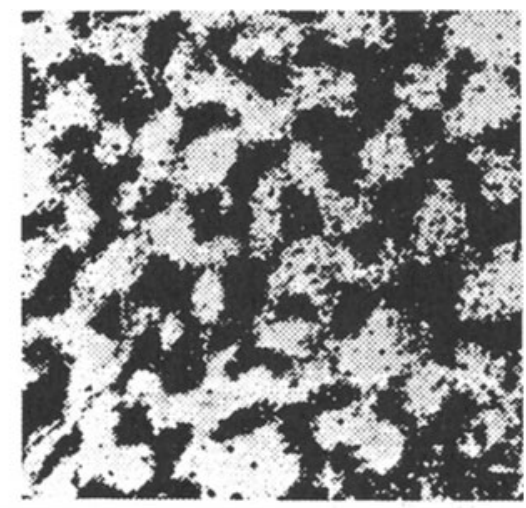

(b)

Figure 3 (a) shows the pattern of ocular dominance in layer IVc of a macaque monkey's primary visual cortex. (b) shows the pattern for the visual cortex of a cat. (a) was adapted from a digitally scanned image of figure 5 of [10], (b) was adapted from figure $7 \mathrm{~b}$ of [11].

\section{Acknowledgements}

We thank Ken Miller, Nick Swindale, Martin Zuckermann, and Thurston Lacalli for helpful discussions. Thanks also to Florence Tam for drawing Figure 1, and Leah Keshet for useful comments on the manuscript. This work was made possible by financial support from NSERC Canada.

\section{References}

[1] M. J. Lyons and L. G. Harrison, Chemical Physics Letters, (accepted for publication).

[2] N. V. Swindale, Proc. R. Soc. Lond. B 208 (1980) 243. 
[3] K.D. Miller, J.B. Keller, and Michael P. Stryker, Science 245 (1989) 605.

[4] D. H. Hubel and T. N. Wiesel, Proc. R. Soc. Lond. B 198 (1977) 1.

[5] J. R. Thomson, Z. Zhang, Wm. Cowan, M. Grant, J. A. Hertz, and M. J. Zuckermann, Physica Scripta, T33 (1990) 102.

[6] H. Haken, Synergetics, 3rd ed. (Springer-Verlag, Berlin, 1983).

[7] L. G. Harrison and M. J. Lyons, Proceedings of the Les Houches Winter Workshop, D. Beysens and G. Forgacs, eds. 1991.

[8] L. G. Harrison and T. C. Lacalli, Proc. R. Soc. Lond. B 202 (1978) 361.

[9] S. Levay, M. P. Stryker, and C. J. Shatz, J. Comp. Neurol. 179 (1978) 223.

[10] S. LeVay, T. N. Wiesel, and D. H. Hubel, J. Comp. Neurol. 191 (1980) 1.

[11] P. A. Anderson, J. Olavarria, and R. C. Van Sluyters, J. Neuroscience 8 (1988) 2183.

[12] A. M. Turing, Phil. Trans. R. Soc. London B 237 (1952) 37. 